\title{
Reconstruction of Attenuation Map Using Discrete Consistency Conditions
}

\author{
Andrei V. Bronnikov
}

\begin{abstract}
Methods of quantitative emission computed tomography require compensation for linear photon attenuation. A current trend in single-photon emission computed tomography (SPECT) and positron emission tomography (PET) is to employ transmission scanning to reconstruct the attenuation map. Such an approach, however, considerably complicates both the scanner design and the data acquisition protocol. A dramatic simplification could be made if the attenuation map could be obtained directly from the emission projections, without the use of a transmission scan. This can be done by applying the consistency conditions that enable us to identify the operator of the problem and, thus, to reconstruct the attenuation map. In this paper, we propose a new approach based on the discrete consistency conditions. One of the main advantages of the suggested method over previously used continuous conditions is that it can easily be applied in various scanning configurations, including fully three-dimensional (3-D) data acquisition protocols. Also, it provides a stable numerical implementation, allowing us to avoid the crosstalk between the attenuation map and the source function. A computationally efficient algorithm is implemented by using the $Q R$ and Cholesky decompositions. Application of the algorithm to computer-generated and experimentally measured SPECT data is considered.
\end{abstract}

Index Terms-Attenuation correction, positron emission tomography (PET), single-photon emission computed tomography (SPECT).

\section{INTRODUCTION}

C OMPENSATION for photon attenuation is one of the major issues in quantitative emission computed tomography. Most state-of-the-art single-photon emission computed tomography (SPECT) and positron emission tomography (PET) systems provide transmission scanning capabilities, enabling us to reconstruct the attenuation map and use it for attenuation correction. This method, however, complicates both the scanner design and the data acquisition protocol. An alternative way to obtain the attenuation map is to reconstruct it directly from the emission data, which would greatly simplify the apparatus and the measurement technique. Two general approaches to this problem have been discussed in literature. The first involves using alternating iterations between the activity image and the attenuation map. Starting from a pioneering paper written by Censor et al. [1], several attempts to apply such methods have been reported [2]-[10]. However, in spite of limited success, methods of simultaneous reconstruction often cause artifacts

Manuscript received September 3, 1999; revised February 1, 2000. The Associate Editors responsible for coordinating the review of this paper and recommending its publication were M. Defrise, and F. J. Beekman.

The author is with KEMA, Arnhem 6800ET, The Netherlands (e-mail: andrei@kema.nl).

Publisher Item Identifier S 0278-0062(00)05309-X. in the form of crosstalk between the activity image and the attenuation map. Another approach involves finding the attenuation map without reconstructing the activity image. In SPECT, the simplest method of doing this is to apply an approximate linear relation that exists between the attenuation map and the data measured at two opposite positions of the scanner [11]. The approximation, however, requires the assumption about relatively low attenuation, which restricts possible applications of such a method. A more general approach was proposed by Natterer [12], who suggested applying the consistency conditions for the range of the attenuated Radon transform to obtain the attenuation map from SPECT-type data. This method has been examined in several papers [13]-[16]. Essentially, the consistency conditions are given in the form of a functional that equals zero on the range of the operator of the problem. This enables us to identify the operator, providing that a function from its range is available. In particular, the attenuation map, which is a parameter of the imaging operator, can be found. An advantage of such an approach is that no information about the unknown activity image is required and no attempt to reconstruct it is made. Certain difficulties appear if the data measured are not in the range of the operator identified because of the noise, discretization errors, and other physical factors that corrupt them. In combination with the ill-posedness of the problem, this causes instability of the solution unless regularization procedures are applied. The idea of regularization is to replace the ill-posed equation of the problem by a nearby well-posed equation. For instance, a stable reconstruction can be provided by such parameterization of the problem that drastically decreases its dimension (see, e.g., [14]). As has recently been shown, this enables us to find the uniform elliptical attenuation distribution that is most consistent with the measured SPECT data [16]. At the same time, it is also possible to restrict a number of the degrees of freedom of the solution by reformulating the problem in a discrete setting. This motivated us to search for a new set of consistency conditions that can be applied to the discretization of the range of the operator.

The approach suggested in this paper is fully based on a discrete representation of the consistency conditions, rather than their continuous description. This has several important consequences. First of all, such an approach can easily be applied with an arbitrary configuration of the scanner, and the use of fully three-dimensional (3-D) data acquisition geometry is an important advantage of our method. Secondly, unlike the continuous method, the discrete method enables us to take into account the influence of various physical factors, such as finite detector resolution, system response, and scatter, which may con- 
siderably improve the accuracy of modeling. The discrete consistency conditions can easily be formulated for both SPECT and PET imaging modalities. Finally, as is shown in this paper, the discrete consistency conditions provide a relatively simple algorithm, whose stability can be controlled by the well-established method of Tikhonov regularization. We consider general mathematical foundations of the consistency conditions and demonstrate a relation existing between continuous and discrete conditions. The discrete consistency conditions are formulated by means of orthogonal projecting onto the orthogonal complement of the column space of the matrix of the problem. We propose to compute the projector by using orthogonal decompositions of the matrix, whereas a corresponding system of nonlinear algebraic equations can be resolved by the Newton method. Such a numerical technique can be classified as an instance of a general optimization approach suggested by Golub and Pereyra [17] for nonlinear least-squares problems whose variables separate. One of the central issues of their approach is the use of a formula for the Frésche derivative of the orthogonal projector [17]. In our previous paper [18], we have proposed a modification of this formula for the case of the identification problem for the attenuated Radon transform and applied the singular value decomposition (SVD) for performing a numerical analysis of the discrete consistency conditions. In this paper, we not only give a broader description and interpretation of the consistency conditions, but also suggest a computationally advanced version of the method, resorting to much more efficient QR and Cholesky decompositions, which makes 3-D reconstruction feasible. The modified Golub-Pereyra formula obtained in paper [18] is used here to compute the Jacobian matrix of the problem. We describe the corresponding algorithm and consider its application to computer-generated and experimental data; for the first time, reconstruction of the attenuation map from emission projections is demonstrated in the framework of cone-beam 3-D SPECT imaging.

\section{THEORY}

\section{A. Continuous Problem}

Consider a general analytical description of the reconstruction problem in SPECT (PET) imaging in the form of

$$
A_{\mu} f=g
$$

where $A_{\mu}$ is a linear integral operator, $f$ is the source function, and $g$ stands for the measured data. The attenuation map $\mu$ is the parameter of the equation. $f$ and $\mu$ are sufficiently smooth functions with finite support. Note that in SPECT reconstruction with two-dimensional (2-D) parallel-beam geometry, operator $A_{\mu}$ stands for the attenuated Radon transform. The reconstruction problem in emission tomography is to find the source function $f$ with known $\mu$ and measured $g$. In the present paper, a problem of determination of the attenuation map $\mu$ from measured data $g$ is considered. After obtaining function $\mu$, the major problem of reconstruction of $f$ can be resolved. One of the methods of obtaining $\mu$ from the $g$ data is to apply the consistency conditions to the range of operator $A_{\mu}$. Let us describe the main principles of this approach.
Let us assume that $g \in H$, where $H$ is a Hilbert space with inner product $\langle,\rangle_{H}$. Let $\bar{H}$ stand for a closed subspace of $H$. Then, any element $g$ can be represented as

$$
g=\bar{g}+g_{\perp}
$$

where $\bar{g} \in \bar{H}$ is an orthogonal projection of $g$ onto $\bar{H}$ and $g_{\perp} \in$ $\bar{H}^{\perp}$ is an orthogonal projection of $g$ onto $\bar{H}^{\perp}$, where $\bar{H}^{\perp}$ is the orthogonal complement to $\bar{H}$; that is, a linear space whose elements are orthogonal to any element of $\bar{H}$. Let $\bar{H} \equiv R\left(A_{\mu}\right)$ and $\bar{H}^{\perp} \equiv R\left(A_{\mu}\right)^{\perp}$, where $R\left(A_{\mu}\right)$ is the range of the integral operator $A_{\mu}$ and $R\left(A_{\mu}\right)^{\perp}$ is the orthogonal complement to this range. The consistency conditions for (1) is then written in the form

$$
g_{\perp}=0 .
$$

Obviously, if (3) holds, $g=\bar{g}$ and (1) can be resolved with respect to $f$. To apply(3), we need to consider the nullspace $N\left(A_{\mu}^{*}\right)$ of the adjoint operator. Because $N\left(A_{\mu}^{*}\right)=R\left(A_{\mu}\right)^{\perp}$, conditions (3) can be interpreted as the requirement of orthogonality of $g$ to $N\left(A_{\mu}^{*}\right)$, which can be written as

$$
\left\langle\psi_{\mu}, g\right\rangle_{H}=0
$$

where $\psi_{\mu}$ is a nonzero solution of a homogeneous linear equation

$$
A_{\mu}^{*} \psi=0
$$

Equation (4) describes the consistency conditions for (1). For the attenuated Radon transform, the ghost functions $\psi_{\mu}$ of the adjoint operator have been found by Natterer, who demonstrated for the first time the use of (4) for reconstruction of function $\mu$ from the $g$ data [12]-[14]. A numerical implementation of such a method requires algebraization of (4) with solving the corresponding system of nonlinear equations by some iterative method.

\section{B. Discrete Problem}

Discretization of (1) provides a system of algebraic equations characterized by matrix $A_{\mu} \in \mathbb{R}^{m \times n}$, where a sampled attenuation map is given by vector $\mu \in \mathbb{R}^{l}$, which plays the role of the parameter of the matrix. In tomography, $A_{\mu}$ is often referred to as the "projection matrix" whose entries can be computed by using a pixel (in 2-D) or voxel (in 3-D) representation of the images (we will refer to voxels throughout this paper). For example, the elements of matrix $A_{\mu}$ for the SPECT problem can be computed as

$$
a_{i j}=\sigma_{i j} e^{-\sum_{k \in K_{i j}} \mu_{k} \sigma_{i k}}
$$

where $\sigma_{i j}$ is the length of intersection of the $i$ th ray with the $j$ th voxel and $K_{i j}$ contains indexes of voxels that are intersected by the $i$ th ray on its segment between the $j$ th voxel and the detector. The source function and the attenuation map can be represented by images with different resolutions: we use $n$ voxels for the activity image and $l$ voxels for the image of the attenuation map. 
A number of equations should be greater than or equal to the number of the unknowns; therefore, we assume

$$
m \geq n+l
$$

which means that matrix $A_{\mu}$ is rectangular.

Because the orthogonal projection can easily be found in linear algebra by using the pseudoinverse, a direct application of (3) is feasible in the discrete setting. Consider a system $A_{\mu} f=g, f \in \mathbb{R}^{n}, g \in \mathbb{R}^{m}$. The linear space $\mathbb{R}^{m}$ can be represented as a direct sum $\mathbb{R}^{m}=R\left(A_{\mu}\right) \oplus R\left(A_{\mu}\right)^{\perp}$ of the column space $R\left(A_{\mu}\right)$ and its orthogonal complement $R\left(A_{\mu}\right)^{\perp}$, meaning that each vector $g \in \mathbb{R}^{m}$ can be written in a unique way as $g=\bar{g}+g_{\perp}$, where $\bar{g} \in R\left(A_{\mu}\right)$ and $g_{\perp} \in R\left(A_{\mu}\right)^{\perp}$. The system is consistent if $g \in R\left(A_{\mu}\right)$, which is equivalent to $g_{\perp}=0$ that coincides with (3). In contrast to the continuous problem, here we can explicitly define the projection $g_{\perp}$ by means of the Moore-Penrose pseudoinverse $A_{\mu}^{+} \in \mathbb{R}^{n \times m}$ of matrix $A_{\mu}$. Note that the pseudoinverse is routinely used in numerical linear algebra to compute the least-squares solution

$$
\tilde{f}=A_{\mu}^{+} g
$$

Using the pseudoinverse matrix, the orthogonal projections onto the column space of $A_{\mu}$ and its orthogonal complement are written in the form

$$
\bar{g}=P_{\mu} g, \quad g_{\perp}=P_{\mu}^{\perp} g
$$

where

$$
P_{\mu}=A_{\mu} A_{\mu}^{+}, \quad P_{\mu}^{\perp}=I-A_{\mu} A_{\mu}^{+}
$$

are $m \times m$ matrices of the orthogonal projectors and $I$ is the identity matrix. Projectors $P_{\mu}$ and $P_{\mu}^{\perp}$ are idempotent matrices; that is, $P_{\mu}^{2}=P_{\mu}$ and $\left(P_{\mu}^{\perp}\right)^{2}=P_{\mu}^{\perp}$. Matrix $P_{\mu}^{\perp}$ is singular. Using the definition of the orthogonal projector, the discrete consistency conditions can be written in the form

$$
P_{\mu}^{\perp} g=0
$$

Equation (11) describes a system of nonlinear equations that can be resolved with respect to vector $\mu$. According to its definition, projector $P_{\mu}^{\perp}$ degenerates into the zero matrix when the pseudoinverse coincides with the right inverse. This is the case when matrix $A_{\mu}$ is a square matrix of a full rank. Thus, the farther the matrix is from the square matrix of a full rank, the stronger condition (11) will be. This emphasizes the role of condition (7), suggesting that the data vector has to have much larger dimension than that of the searched vector. The question of how large it must be was studied in [18], where the singular values of the Jacobian matrix of (11) were computed for fixed $n=l$ and variable values of $m$. Using computer-generated data, it was shown that for some particular problem, the choice $m=1.5 n$ caused poor conditioning of the problem, whereas the case $m=3 n$ demonstrated satisfactory behavior of the singular values, which, however, did not improve much for any $m>3 n$.

The discrete formulation of the problem permits a discrete version of (4) to be found. This provides a connection with the continuous consistency conditions. Observe that the requirement of orthogonality of $g$ to $R\left(A_{\mu}\right)^{\perp}$ can be written as

$$
\psi_{\mu}^{\top} g=0
$$

where $\psi_{\mu} \in \mathbb{R}^{m}$ is a vector that is orthogonal to each column of matrix $A_{\mu}$; that is

$$
A_{\mu}^{\top} \psi_{\mu}=0
$$

Equations (12) and (13) are discrete counterparts of (4) and (5), so that vector $\psi_{\mu}$ can be interpreted as a discrete counterpart of the ghost function used in the continuous consistency conditions. Because all vectors satisfying (13) form a complete orthonormal basis for $R\left(A_{\mu}\right)^{\perp}$, vector $\psi_{\mu}$ is one of the vectors of such a basis. Therefore, (12) expresses nothing but a single equation from system (11). Thus, rows of projector matrix $P_{\mu}^{\perp}$ can be interpreted as discrete counterparts of the ghost functions used in the continuous consistency conditions.

Equation (12) has a minor independent role because the use of a single equation would require minimization of a function of $l$ variables instead of solving a system of nonlinear equations as for the conditions given in the form of (11). This would require computation of the Hessian matrix, whereas to resolve (11), it would be sufficient to compute the Jacobian matrix. Also, analytical differentiation of a single row may prove to be a problem, in contrast with differentiation of the entire projector. This suggests using (11) for numerical implementation. A corresponding algorithm is described in the next section.

\section{METHOD}

\section{A. Computation of the Projector}

To implement the discrete consistency conditions, the projector $P_{\mu}^{\perp}$ has to be computed. This can be done in different ways. The most straightforward approach is to use the pseudoinverse matrix as in (10), which is perhaps the worst thing to do because of cumbersome and lengthy computations. The use of orthogonal decompositions provides much faster solutions. For instance, the Gram-Schmidt orthogonalization can be used to explicitly compute a basis for the column space of the matrix. This method requires $m n^{2}$ flops. Another approach is to apply the SVD, where the left singular vectors form an orthonormal basis for the column space; this algorithm has a complexity of $7 m n^{2}-n^{3}$ flops. A good alternative is provided by the $\mathrm{QR}$ decomposition of the matrix. The $\mathrm{QR}$ decomposition requires a minimal number of operations, which can be estimated as $m n^{2}-n^{3} / 3$ flops. In the $\mathrm{QR}$ decomposition, the matrix is represented as

$$
A_{\mu}=Q\left(\begin{array}{c}
R \\
0
\end{array}\right)
$$

where $Q \in \mathbb{R}^{m \times m}$ is the orthogonal matrix and $R$ is upper triangular with nonnegative diagonal elements. Matrix $Q$ can be partitioned as $Q=\left(Q_{1}, Q_{2}\right)$, where $Q_{1} \in \mathbb{R}^{m \times n}$ and $Q_{2} \in$ $\mathbb{R}^{m \times(m-n)}$. The columns of $Q_{1}$ and $Q_{2}$ form orthonormal bases for the column space of matrix $A_{\mu}$ and its complement. Thus, 
the pseudoinverse matrix is $A_{\mu}^{+}=R^{-1} Q_{1}^{\top}$, and the projectors are

$$
P_{\mu}=Q_{1} Q_{1}^{\top}, \quad P_{\mu}^{\perp}=Q_{2} Q_{2}^{\top} .
$$

\section{B. Reconstruction Algorithm}

A solution of the nonlinear system of (11) can be found by the iterative search algorithm such that

$$
\mu^{(k)}=C\left(\mu^{(k-1)}-\Delta \mu\right)
$$

where $\Delta \mu \in \mathbb{R}^{l}$ is the step size of the algorithm. $C$ denotes a nonlinear operator that enables us to impose certain constraints on the attenuation map. For example, in the case of the nonnegativity of function $\mu$, the $j$ th element of vector $C \mu$ can be represented by

$$
(C \mu)_{j}= \begin{cases}\mu_{j}, & \text { if } \mu_{j}>0 \\ 0, & \text { otherwise. }\end{cases}
$$

Information about values of the attenuation coefficient for different tissues can be incorporated into the algorithm in a similar way. The initial estimate $\mu^{(0)}$ can be set to zero or an a priori known approximation. In each step $k$, matrix $A_{\mu}$ is computed by formula (6), its QR decomposition (14) is performed, and vector $\Delta \mu$ of the step size is obtained by the Newton method, which requires solving equation

$$
J_{\mu} \Delta \mu=g_{\perp}
$$

where

$$
g_{\perp}=Q_{2} Q_{2}^{\top} g
$$

and $J_{\mu}$ is the Jacobian matrix of the problem. The Jacobian matrix can be found as

$$
J_{\mu}=-Q_{2} Q_{2}^{\top} B_{\mu}
$$

where

$$
B_{\mu}=\left(\frac{\partial A_{\mu}}{\partial \mu_{1}} \tilde{f} \frac{\partial A_{\mu}}{\partial \mu_{2}} \tilde{f} \cdots \frac{\partial A_{\mu}}{\partial \mu_{l}} \tilde{f}\right)
$$

is an $m \times l$ matrix and $\mu_{j}$ stands for the $j$ th element of vector $\mu$ [18]. Using (6), the entries of matrix $B_{\mu}$ are computed as

$$
b_{i j}=-\sigma_{i j} \sum_{k \in K_{i j}} \tilde{f}_{k} a_{i k}
$$

where $K_{i j}$ contains indexes of voxels that are intersected by the $i$ th ray on its segment between the $j$ th voxel and the boundary of the support of the source function on the side opposite to the detector.

A few remarks on the algorithm are in order. First, note that the Jacobian matrix contains the projector $Q_{2} Q_{2}^{\top}$. Because $Q_{2}$ is an $m \times(m-n)$ column-orthogonal matrix, $Q_{2} Q_{2}^{\top}$ is a $m \times m$ matrix with rank $m-n$, which makes the Jacobian matrix singular by its construction. This reflects the fact that determination of the attenuation map from emission projections is a severely ill-posed problem. To find a stable solution of such a problem, a regularization is required. We discuss the regularization method in the next paragraph. Also, note that the computation of the Jacobian matrix involves finding the least-squares solution $\tilde{f}$. In this way, the algorithm suggested provides reconstruction of the source function as well. At the same time, the activity distribution is represented here via $g$ and $\mu$ as in (8), and the residual $g_{\perp}$ of the method is minimized with respect to vector $\mu$ alone, which makes our approach different from the methods of simultaneous reconstruction of $f$ and $\mu$, where both vectors explicitly contribute to minimization of the residual. In combination with the regularization, this allows us to avoid the crosstalk between the source function and the attenuation map. It should be pointed out that our algorithm may not provide an optimal reconstruction of $f$, because the choice of its parameters is aimed at stable reconstruction of the attenuation map in the first place. However, after obtaining the attenuation map, the source function can be found by any method that is better suited for this. For instance, as shown in Section IV, the Tikhonov method with an optimally chosen regularization parameter can also be used. Another interesting observation is that matrix $B_{\mu}$ has to have zero $i$ th row if $g_{i}=0$, because all corresponding $\tilde{f}_{k}, k \in K_{i j}$ have to be zero. This information can be used to reduce the size of the problem by removing zero rows of matrix $B_{\mu}$ and forming vector $g$ with nonzero data. This has a clear practical explanation: no information on the attenuation map is available in the case of zero emission.

\section{Regularization}

In each step of the algorithm, two systems of algebraic equations have to be resolved. First, the least-squares solution $\tilde{f}$ has to be computed, and second, a solution of system (17) has to be found. We have studied several different methods for solving these problems. The most straightforward method would be to apply an iterative technique similar to the algebraic reconstruction technique (ART). However, such methods failed to provide sufficient quality of the solution. The reason of that was poor conditioning of the Jacobian matrix, which is singular because of the projector. This caused a slow convergency or instability of iterative methods. At the same time, implementation of regularization procedures presents certain difficulties in such approaches. The use of combined algorithms, where $\tilde{f}$ is approximated by a fast iterative method and system (17) is resolved by an orthogonal decomposition of its matrix, did not give a desired result, because the iterative method failed to provide the accuracy required for computing the entries of the Jacobian matrix. It has been found that the Tikhonov regularization method can provide a sufficient quality of the solution.

The idea of Tikhonov regularization is to approximate the least-squares solution in a stable way. This can be achieved by minimization of the following functional:

$$
\left\|g_{\perp}-J_{\mu} \Delta \mu\right\|^{2}+\alpha\left\|L_{1} \Delta \mu\right\|^{2}
$$

where $L_{1}$ is an $l \times l$ matrix approximating the Laplacian and $\alpha>0$ is the regularization parameter. The second term of functional (20) is called the "stabilizing functional." Its role is to suppress instabilities in the solution, providing a certain degree of smoothness that is controlled by the value of the regularization parameter. The regularization parameter has to be chosen 


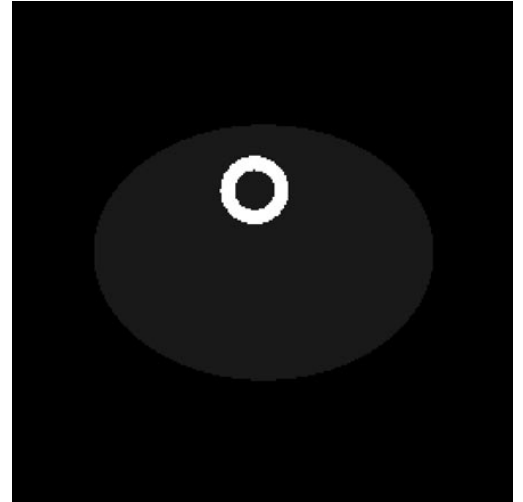

(a)

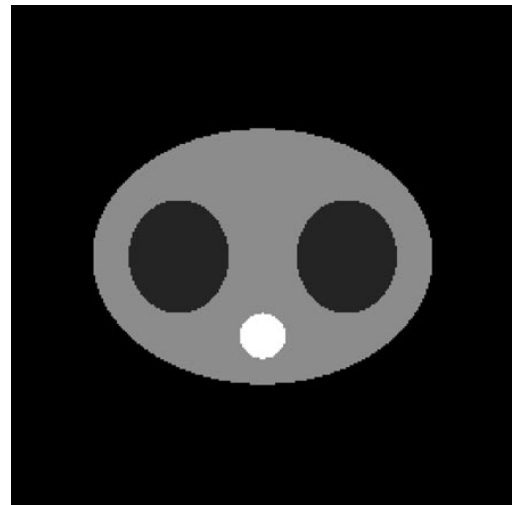

(b)

Fig. 1. (a) The numerical phantom of the source and (b) the attenuation map, simulating cardiac SPECT imaging

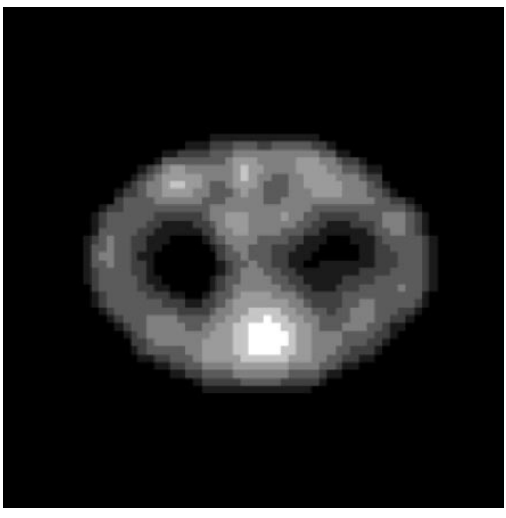

(a)

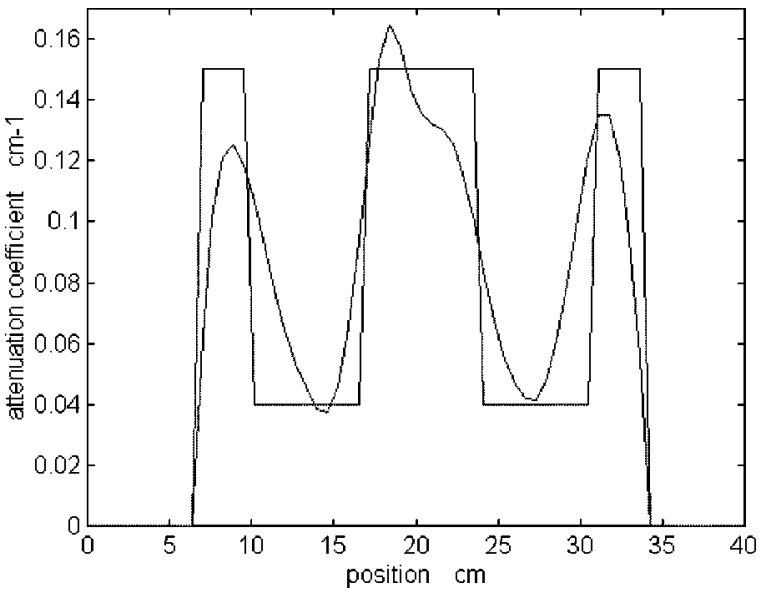

(b)

Fig. 2. Reconstruction of the attenuation map from noisy emission projections computed for the numerical phantom: (a) the reconstructed image of the attenuation map and (b) the attenuation coefficient versus the position across the numerical phantom and its reconstruction. The reconstruction was obtained with parameters $\alpha=0.004$ and $\beta=0.01$; the initial guess $\mu^{(0)}$ was zero.

in accordance with a priori level of the error in the data. However, because of the large size of the problem, a procedure for estimation of the regularization parameter is not feasible here. We assume that the value of the regularization parameter can be found by trial and error using computer simulations of smaller problems. The minimizer of functional (20) is given as a solution of system

$$
\mathcal{J}_{\mu}^{\alpha} \Delta \mu=J_{\mu}^{\top} g_{\perp}
$$

where $\mathcal{J}_{\mu}^{\alpha}=\alpha L_{1}^{\top} L_{1}+J_{\mu}^{\top} J_{\mu}$ is an $l \times l$ symmetrical matrix. The choice of the regularization parameter has to ensure that matrix $\mathcal{J}_{\mu}^{\alpha}$ is positive definite. The most efficient method of solving systems with positive-definite symmetrical matrices is based on the Cholesky factorization. We apply the Cholesky factorization $\mathcal{J}_{\mu}^{\alpha}=R^{\top} R$, where $R$ is an upper-triangular matrix. System $R^{\top} R \Delta \mu=J_{\mu}^{\top} g_{\perp}$ can be efficiently resolved, providing the regularized solution $\Delta \mu_{\alpha}$, which is used to update the vector of the attenuation map in (16). The same approach is used to approximate the least-squares solution $\tilde{f}$. First, system

$$
\left(\beta L_{2}^{\top} L_{2}+A_{\mu}^{\top} A_{\mu}\right) f=A_{\mu}^{\top} g
$$

with a positive-definite symmetrical matrix is formed. (Here, $L_{2}$ is an $n \times n$ matrix approximating the Laplacian and $\beta>0$ is the regularization parameter). Then, this system is resolved by applying the Cholesky factorization, and the regularized solution $f_{\beta}$ is used in (19) instead of the least-squares solution. Thus, in each iteration of the algorithm, we compute one QR decomposition of $m \times n$ matrix $A_{\mu}$ and two Cholesky factorizations: first we compute the factorization of an $n \times n$ matrix to find vector $f_{\beta}$ and then the factorization of $l \times l$ matrix $\mathcal{J}_{\mu}^{\alpha}$ to resolve system (21). After the attenuation map is reconstructed, the final reconstruction of the source function can be made by solving system (22).

\section{RESULTS}

\section{A. Computer Simulations}

To evaluate the approach, computer simulations of SPECT imaging were carried out with a mathematical torso phantom. In the first set of experiments, a 2-D problem with a relatively high resolution of the image was studied. The second study was devoted to 3-D cone-beam reconstruction. Let us first consider the $2-\mathrm{D}$ problem. The numerical phantom simulated a cross section through the thorax. The source was modeled as a ring representing activity in the heart and an ellipse representing background activity in the human body [see Fig. 1(a)]. The background activity was 10 times less than was the activity in the 


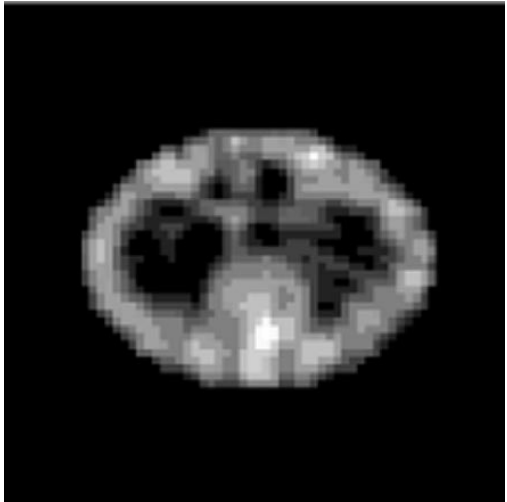

(a)

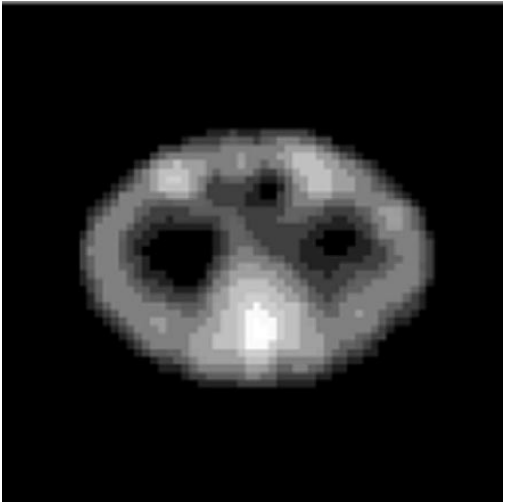

(b)

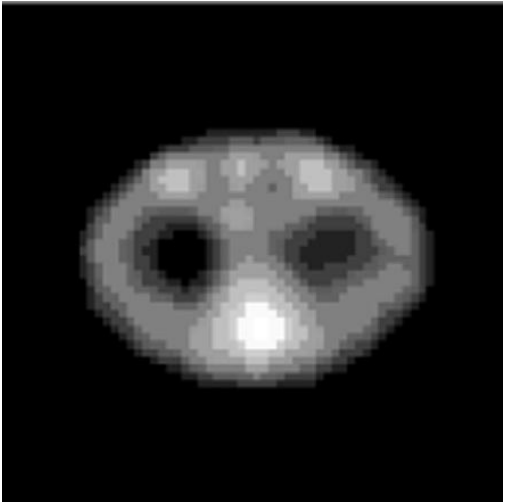

(c)

Fig. 3. Reconstruction of the attenuation map with different regularization parameters: (a) $\alpha=0.0005, \beta=0.01$; (b) $\alpha=0.004, \beta=0.0005$; (c) $\alpha=0.008$, $\beta=0.01$

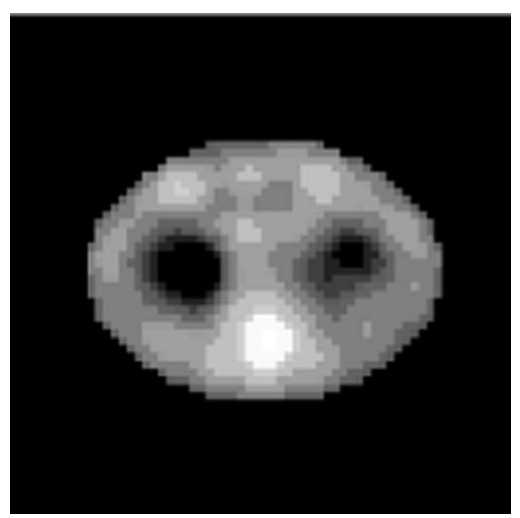

(a)

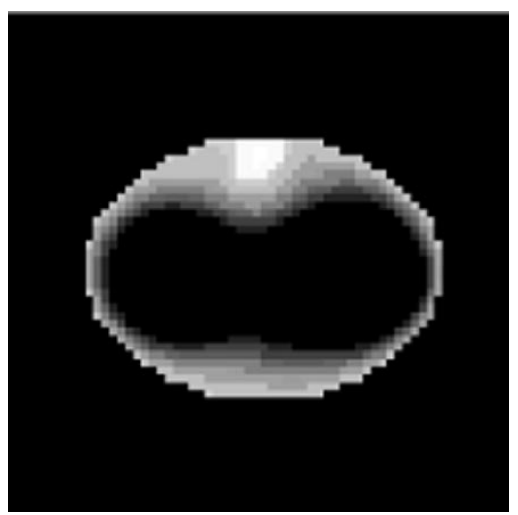

(b)

Fig. 4. Reconstruction of the attenuation map with different initial approximations: (a) all components of vector $\mu^{(0)}$ are set to 0.1 ; (b) all components of vector $\mu^{(0)}$ are set to 0.3 . The reconstructions were made with parameters $\alpha=0.004, \beta=0.01$, and $k=3$.

heart. The attenuation map was represented by two ellipses associated with the lungs, a small circle for the spine bone and a large ellipse for the body contour [see Fig. 1(b)]. The major axis of the ellipse representing the body was $30 \mathrm{~cm}$. The attenuation coefficient was equal to zero for air, $0.04 \mathrm{~cm}^{-1}$ for the lungs, $0.15 \mathrm{~cm}^{-1}$ for the soft tissue, and $0.27 \mathrm{~cm}^{-1}$ for the spine. For characteristic functions of the ellipse, the linear integrals can be evaluated analytically. We applied such a formula to compute emission projections of the phantom. Sixty views over $360^{\circ}$ were simulated with 64 pixels in each view. To more accurately represent a practical situation, Poisson noise was added to the data, so that the NMSE of the projections was about $8 \%$. The total number of counts in the projection data was $\sim 3 \times 10^{5}$. No photon scatter was simulated. The body contour was assumed to be known. Precisely, the support of the source function was computed by using positions of nonzero data. This procedure provided a mask that was used for both the source function and the attenuation map. The images were sampled with $64 \times 64$ pixels, but there were only $n=l=1177$ pixels within the support of the functions to be reconstructed. All zero line integrals were omitted, so that the data vector had the dimension of $m=2492$.

Fig. 2(a) shows the attenuation map reconstructed after using $k=3$ iterations of the algorithm. The reconstruction required about $10 \mathrm{~min}$ on a $\mathrm{PC}$ with a Pentium II $450-\mathrm{MHz}$ processor. Parameters $\alpha=0.004$ and $\beta=0.01$ were used. The initial guess for the attenuation map was zero. As seen from Fig. 2(a), the use of regularization provides a smooth function of the attenuation map, but at the same time, the lungs, the spine, and the body contour are clearly seen and the reconstructed image corresponds well to the phantom. No crosstalk between the attenuation map and the source function is visible. To make a more quantitative comparison, we have depicted the profiles across the phantom and its reconstruction in Fig. 2(b). Here, we can see that the values of the attenuation map are reconstructed satisfactorily. To investigate the influence of the regularization parameters and the initial approximation, several computer experiments were made. Fig. 3 depicts the results obtained in an experiment with three different sets of the regularization parameters. Here, we can see that the decrease of parameter $\alpha$ reduces the stability of the reconstruction in the heart region [Fig. 3(a)]. This instability is similar to the crosstalk of the images reported for the similar phantom by Manglos and Young [3]. The instability in the heart region also develops for the smaller value of parameter $\beta$ [compare Fig. 3(b) and Fig. 2(a)]. Fig. 3(c) shows the case in which both parameters are large enough to provide the stable reconstruction with no crosstalk. Comparing images in Fig. 3, we may conclude that the stability of reconstruction in the heart region is strongly 


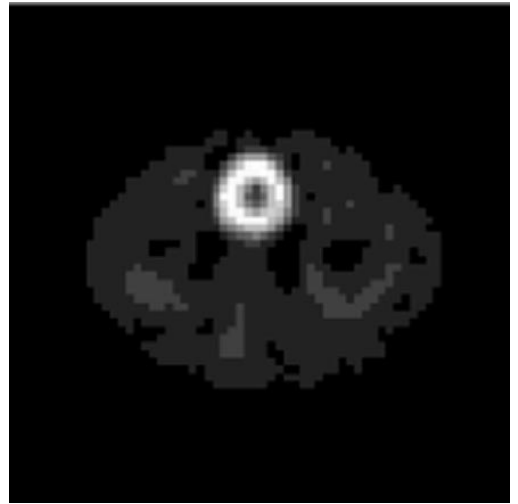

(a)

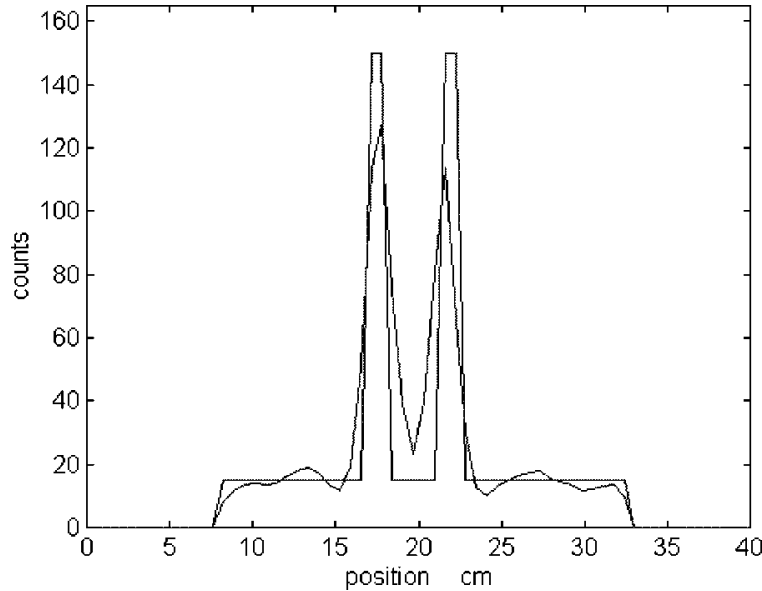

(b)

Fig. 5. Function $f_{\beta}(\beta=0.01)$, which was used for computing the Jacobian matrix in the final step of the algorithm: (a) the grayscale image; (b) the intensity profiles across the numerical phantom and function $f_{\beta}$.

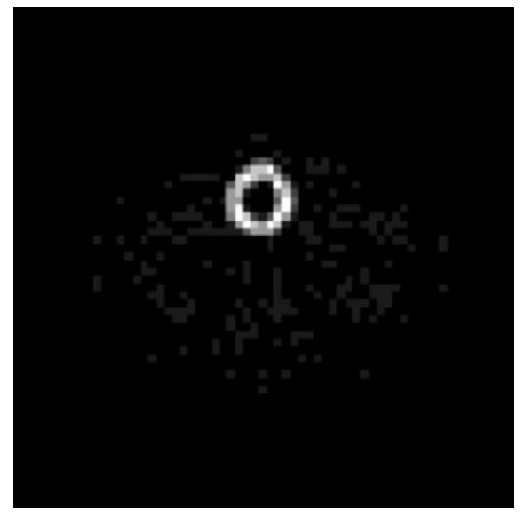

(a)

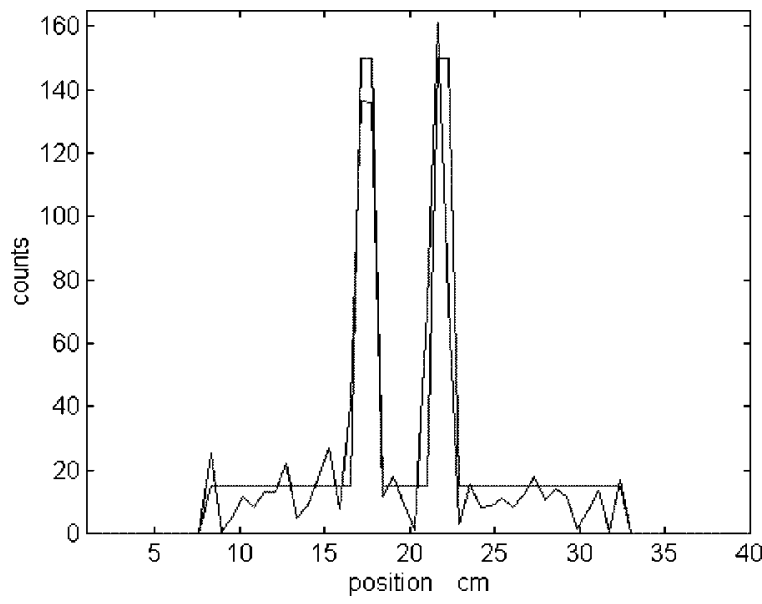

(b)

Fig. 6. Reconstruction of the activity distribution using attenuation correction provided by the attenuation map as in Fig. 2: (a) the reconstructed image of the activity distribution; (b) the intensity profiles across the numerical phantom and its reconstruction. The reconstruction was made by applying the Tikhonov regularization method with parameter $\beta=0.00001$.

dependent on the choice of both regularization parameters. The values of the parameters must not be too small. At the same time, excessively large values of the regularization parameter may introduce errors in quantitative determination of the attenuation coefficients. Thus, the strategy for the choice of the regularization parameters must include a tradeoff between the error in quantification of the attenuation map and the instability in the form of the crosstalk of the images; the regularization parameters can be found by trial and error. In the next computer experiment, the influence of the initial guess was considered. First, all components of vector $\mu^{(0)}$ were set to 0.1 . Using the same parameters of the algorithm as for the reconstruction with the zero initial guess [Fig. 2(a)], we obtained the image depicted in Fig. 4(a). Here, we can see that the reconstruction is stable and the values of the attenuation coefficients are slightly improved as compared with the case of the zero initial guess. Setting the components of the initial vector to 0.3, the image shown in Fig. 4(b) was obtained. As seen from this figure, the algorithm has diverged for this choice of the initial guess. A possible explanation of this can be the divergence of the Newton method in the case of a relatively large residual of the problem.

Although the main goal of this paper is to describe a method for determination of the attenuation map, it is interesting to see how estimation of the attenuation map influences reconstruction of the activity image. First of all, let us demonstrate the activity distribution that was found as a "byproduct" of our algorithm. Fig. 5 demonstrates function $f_{\beta}$, which was used for computing the entries of the Jacobian matrix in (19) of the algorithm. This function was obtained in the final step of the algorithm, and therefore, it corresponds to the attenuation map as in Fig. 2(a). We can see that the use of the Tikhonov regularization method provided a relatively smooth reconstruction with accurate estimation of the background activity. The level of intensity in the heart region is, however, lower than that of the phantom. Nevertheless, this function allowed us to compute a stable reconstruction of the attenuation map with no crosstalk. It should be pointed out that having the attenuation map, any reconstruction method can be used to find the activity image. Depending on the method and its parameters, different activity images can 


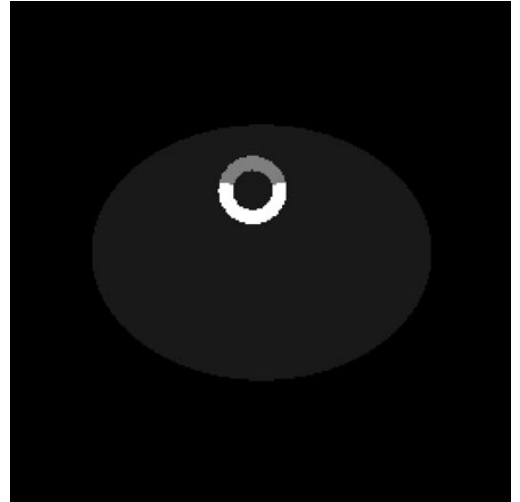

(a)

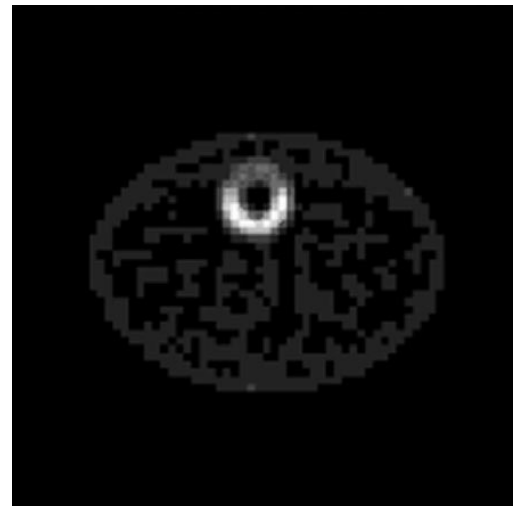

(c)

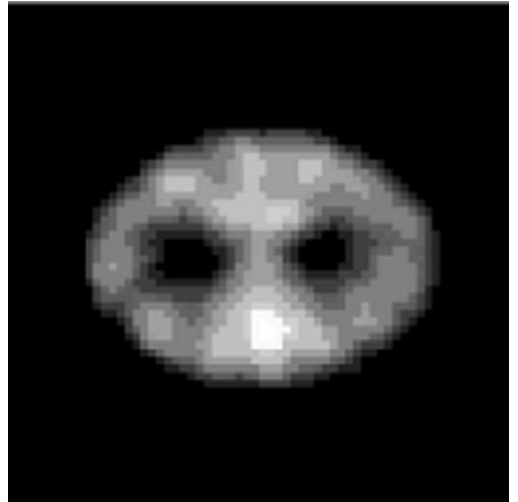

(b)

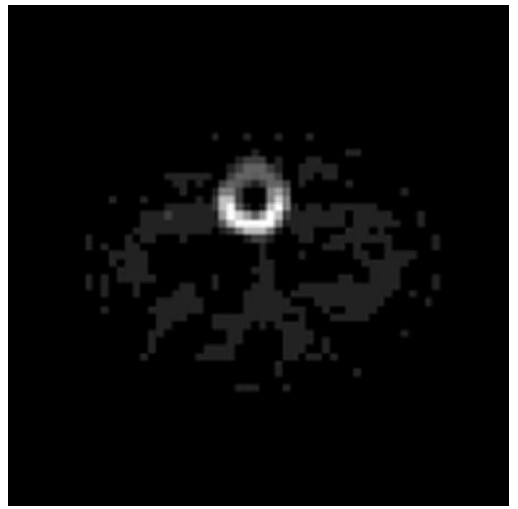

(d)

Fig. 7. Results for the numerical phantom, simulating the myocardial defect: (a) the phantom of the activity image; (b) reconstruction of the attenuation map using discrete consistency conditions; (c) reconstruction of the activity image with attenuation correction using the true attenuation map as in Fig. 1(b); (d) reconstruction of the activity image with attenuation correction using the estimated attenuation map as in Fig. 7(b).

be found for the same attenuation map. This is illustrated in Fig. 6, where the results of applying the Tikhonov regularization method based on (22) are shown. The value of the regularization parameter $\beta$ was here 1000 times smaller than that for the reconstruction as in Fig. 5. Therefore, good spatial resolution was achieved. As seen from Fig. 6(b), the peaks of the intensity in the heart region are resolved well and the values of the intensity are close to those of the phantom. Note that the use of the small value of the regularization parameter caused the amplification of noise in the background area. The profiles across the phantom and the reconstructed image [Fig. 6(b)] show that satisfactory compensation for attenuation has been achieved. The absolute error in determination of activity in the heart area is in the range of $8 \%$, which corresponds well to the discrepancy of the data.

To compare our results with those obtained by using other methods, we have considered another numerical phantom, which was slightly different from the previous one, comprising the myocardial defect. The activity image of this phantom is similar to that in [6]. The heart was represented by the ring with two levels of intensity: 150 counts/pixel for the normal myocardium and 75 counts/pixel for the myocardial defect. The attenuation map was as in Fig. 1(b). For reconstruction of the attenuation map, we used the same parameters as for the reconstruction presented in Fig. 2(a). Despite certain differences in the reconstruction of the attenuation map for the phantom with and without the myocardial defect [compare

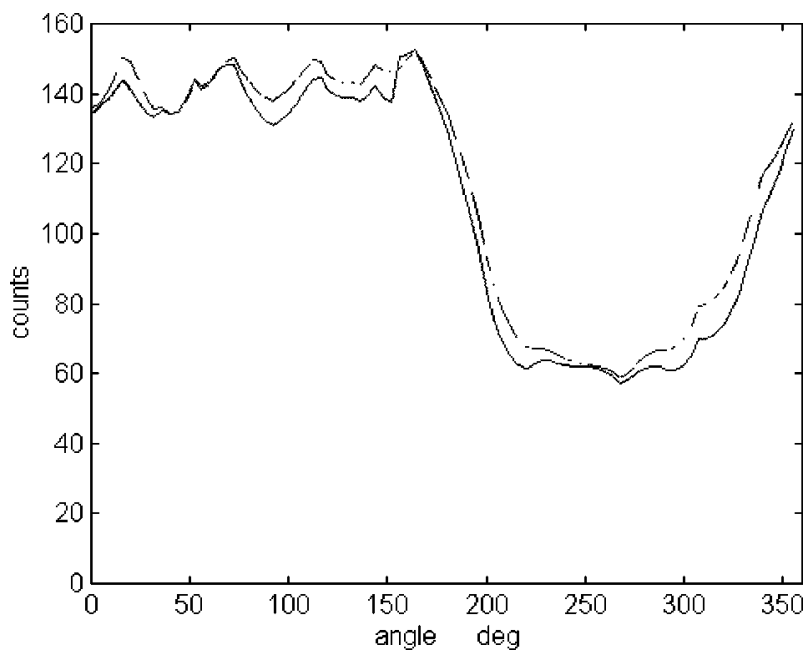

Fig. 8. Circumferential myocardial profiles of the reconstructions obtained with the true (dashed lines) and estimated attenuation maps.

Figs. 7(b) and 2(a)], the reconstruction is stable and no trace of the crosstalk is visible. We remark that our reconstruction was obtained under far fewer conditions than in [6], in which measurements with two different energies were required and $a$ priori information of constant attenuation was used to suppress the crosstalk by assigning large penalties to the cost function in the heart region. The comparison with the activity reconstruction based on the true attenuation map is given in Fig. 7(c) and 

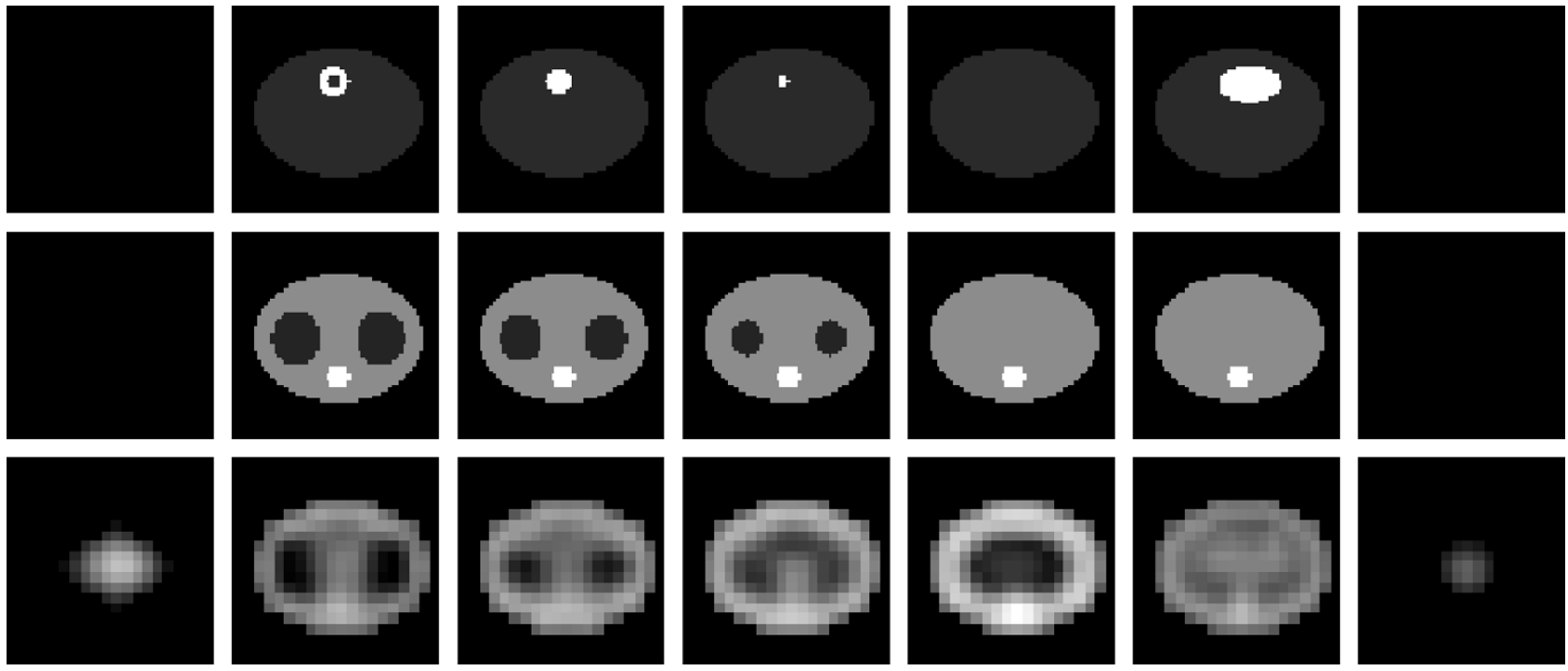

Fig. 9. Simulation of 3-D cone-beam SPECT imaging. The upper row: seven representative axial slices of the 3-D numerical phantom of activity. The middle row: axial slices of the 3-D numerical phantom of attenuation. The lower row: reconstruction of the attenuation map from cone-beam SPECT emission projections.

(d). Fig. 7(c) shows the source function reconstructed with the true attenuation map by the Tikhonov regularization method with $\beta=0.0005$. The same method was used to reconstruct the image based on the estimated attenuation map [Fig. 7(d)]. Both images correspond well to the phantom and have relatively small differences. The image obtained with the true attenuation map has slightly more homogeneous background activity, especially near the boundary of the object. This can be explained by the fact that the true attenuator has the abrupt edge, whereas the estimated attenuation map has a roll-off of the values near its boundary. To make a more quantitative comparison, we used the circumferential myocardial profiles as shown in Fig. 8. Here, we can see that both reconstructions coincide well, having a deviation of no more than $10 \%$. The activity image obtained with the estimated attenuation map has slightly lower values than does the image based on the true attenuation map. This can be explained by the fact that the estimated attenuation map has lower values because of the regularization. Nevertheless, Figs. 7 and 8 demonstrate that the suggested method provides results similar to those obtained by using the true attenuation map.

One of the most attractive features of the discrete consistency conditions is that they can easily be applied for fully 3-D imaging. We have simulated an experiment with 3-D cone-beam SPECT imaging using a circular scan. In this experiment, the focal point of the converging collimator was rotated with the radius of rotation of $40 \mathrm{~cm}$. The size of the 2-D detector was $40 \times 40 \mathrm{~cm}$, and the focal length of the collimator was $60 \mathrm{~cm}$. The projection was sampled with $64 \times 64$ pixels, and 60 views over $360^{\circ}$ were computed. To reduce the size of the problem, we used a coarser grid for reconstruction and applied a mask computed by using nonzero values of the projections. This gave $n=l=2946$ and $m=15978$, so that about $350 \mathrm{Mb}$ of computer memory was required. Fig. 9 demonstrates seven representative axial slices of the 3-D phantom and its reconstruction. The upper row in Fig. 9 shows the phantom of the activity distribution. Two hot spots were located in the heart and the liver.

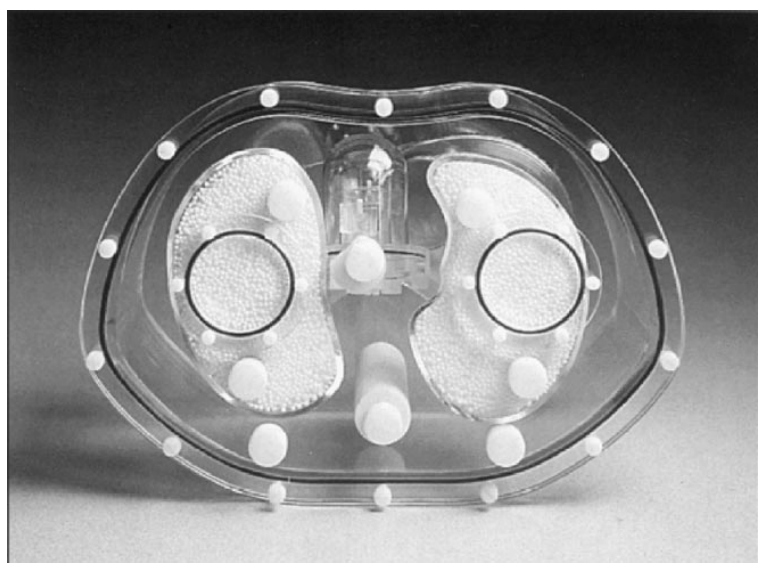

Fig. 10. The Jasczack anthropomorphic torso phantom. The photograph is by courtesy of Data Spectrum Company.

The background activity was 10 times less than was the activity in the hot spots. The middle row represents the 3-D attenuation map. Here, we see the lungs, the spine, and the area of the tissue around the liver. The attenuation coefficients of the phantom were the same as those of the 2-D phantom. The data were reconstructed by using the algorithm suggested with $k=2$ iterations and $\alpha=0.01, \beta=0.05$. The initial guess for the attenuation map was zero. The lower row in Fig. 9 shows the reconstruction of the attenuation map from simulated emission projections. As seen from the first and the last image in the row, nonzero values were reconstructed in the area where the actual attenuation map was zero. This can be explained by the influence of the oblique rays in the cone-beam geometry, which suggests that different slices in the reconstructed image influence each other. We can also see that the reconstruction of the attenuation map is strongly dependent on the configuration of the source. For instance, the slices through the heart are similar to the reconstruction obtained in the case of parallel-beam geometry. Here, we see the area of the lungs and the area of the constant attenuation. The attenuation coefficient is close to that of 


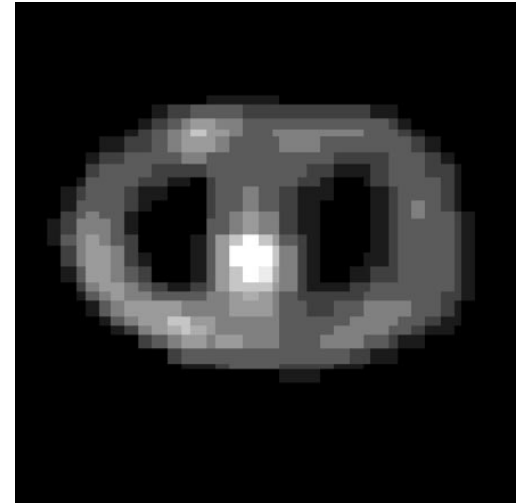

(a)

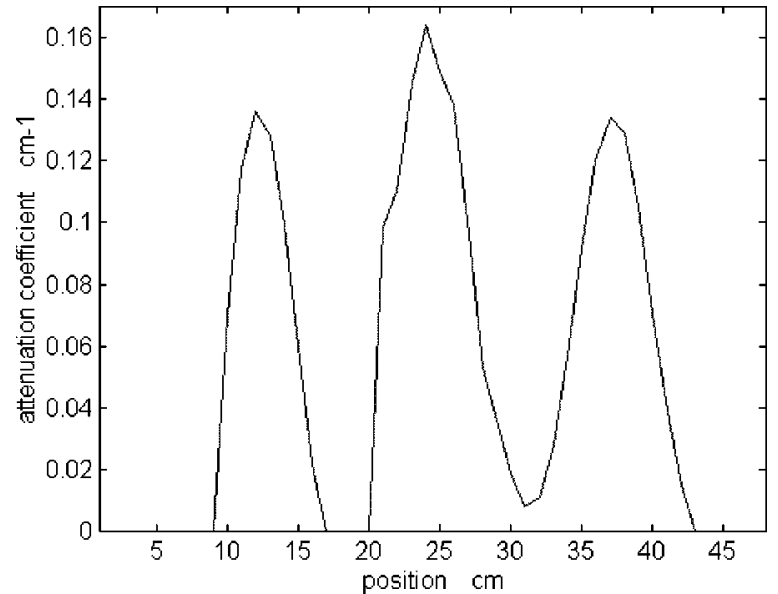

(b)

Fig. 11. Reconstruction of the attenuation map of the anthropomorphic torso phantom: (a) the reconstructed image of the attenuation map; (b) the attenuation coefficient versus the position across the reconstruction of the attenuation map.

the phantom. However, the quality of reconstruction deteriorates in the slices where no sources are present, except those of the background or a single point source. Such slices are shown in the fourth and fifth images from the left-hand side in the lower row. In the slice with the point source, the attenuation map contains an error within the area of the source. The values of the attenuation coefficient are too small there. This problem is much worse in the next slice (the image in the middle of the lower row). Here, we see a large error in the reconstruction of the attenuation map. It is interesting to note that this image has a symmetrical pattern (except the area of the spine, which was slightly emphasized). This kind of error can be explained by the fact that the problem has no unique solution in the case of symmetrical functions $f$ and $\mu$ [13]. Both functions are almost symmetrical here, which might cause the error in the given slice and deteriorate the neighboring areas as well. For instance, in the next slice, we can see that in spite of the presence of an asymmetrical source, the reconstruction of the attenuation map still contains certain artifacts with a symmetrical structure. Nevertheless, the presence of a strong source (the liver) provides a satisfactory reconstruction of the average value of the attenuation coefficient, showing no crosstalk in the form of low attenuation, which is one of the advantages of the suggested algorithm.

\section{B. Experimental Data}

In the last study, we used experimental data obtained at the Utrecht University Hospital by applying a SPECT measurement system to the Jasczack anthropomorphic torso phantom [see Fig. 10]. The phantom contains different features, such as lungs and spine, and enables us to simulate activity in the heart, liver, and tissue. The phantom was filled with Tc-99m; the amount of activity in the left ventricle was $18 \mathrm{MBq}$, and the background contained $84 \mathrm{MBq}$. A standard SPECT imaging system was used to acquire 128 projections over $360^{\circ}$ with a resolution of 64 pixels. The emission and transmission acquisition times were 25 and 24 seconds per view, respectively. More details of the experiment and parameters of the phantom are given by Beekman et al. [19]. The emission projections were used to reconstruct the attenuation map and the activity image by using the algorithm suggested. No scatter correction was applied. The resolution of the reconstructed image was $64 \times 64$ pixels, $\alpha=\beta=0.01$, and $k=3$. The initial guess for the attenuation map was zero. The reconstruction time was about $25 \mathrm{~min}$. Note that the reconstruction time rapidly decreases for coarser images. For instance, reconstruction of a $32 \times 32$ image required about $30 \mathrm{~s}$. In such a case, the time required for reconstruction of the attenuation map by the algorithm suggested is comparable to the transmission data acquisition time. Generally, the reconstruction time can be considerably shortened by optimizing the source code of the algorithm and using a faster computer. The reconstruction of the attenuation map is shown in Fig. 11(a). Here, we can see that the body contour and the lungs are reconstructed well. At the same time, the spine was not reconstructed and the algorithm produced a noticeable error near the center of the image. Nevertheless, as seen in Fig. 11(b), the values of the attenuation map are close to the expected ones, which can secure satisfactory compensation for attenuation. This is demonstrated in Fig. 12(a), where reconstruction of the activity image based on the estimated attenuation map is depicted. These results can be compared with an image obtained with a transmission-based attenuation map [Fig. 12(b)]. Both images were reconstructed by the Tikhonov regularization method with parameter $\beta=0.0005$. To minimize a possible influence of the errors in the center of the estimated attenuation map, the activity reconstructions were made on a $180^{\circ}$ sector of the projection data, using 64 views. Fig. 12(a) and (b) demonstrate that sourceless attenuation correction provides the image comparable to that of the algorithm using the attenuation map obtained from transmission measurements. To evaluate the discrepancy between the images, a difference image, which is the magnitude of the difference of the pixel values of the images, was computed (Fig. 13). As we can see in Fig. 13, the difference of the images is maximal along the boundary of the object. This can be explained by the fact that the estimated attenuation map has a large error near the boundary of the attenuator. Also, there is a certain discrepancy in the heart region, which is, however, relatively small compared with the values of the activity in this area. As seen from the intensity profiles (Fig. 14), the peaks of activity in the heart coin- 


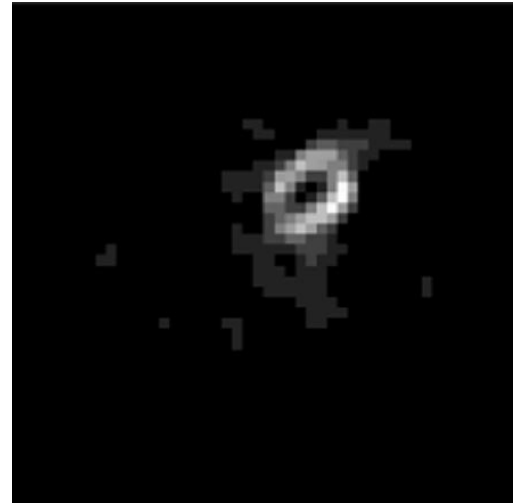

(a)

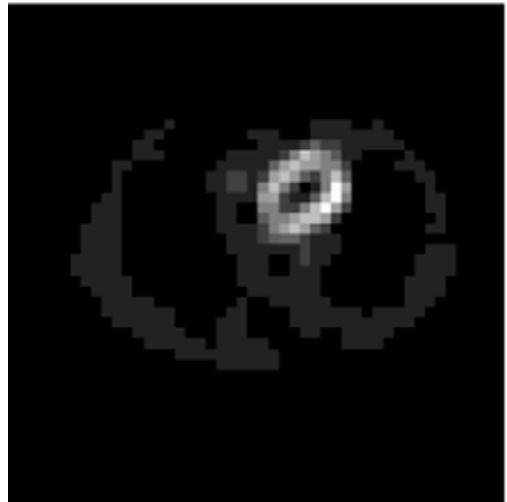

(b)

Fig. 12. Reconstructions of the activity image of the anthropomorphic torso phantom: (a) reconstruction using the attenuation map estimated from SPECT emission projections; (b) reconstruction using transmission-based attenuation correction.

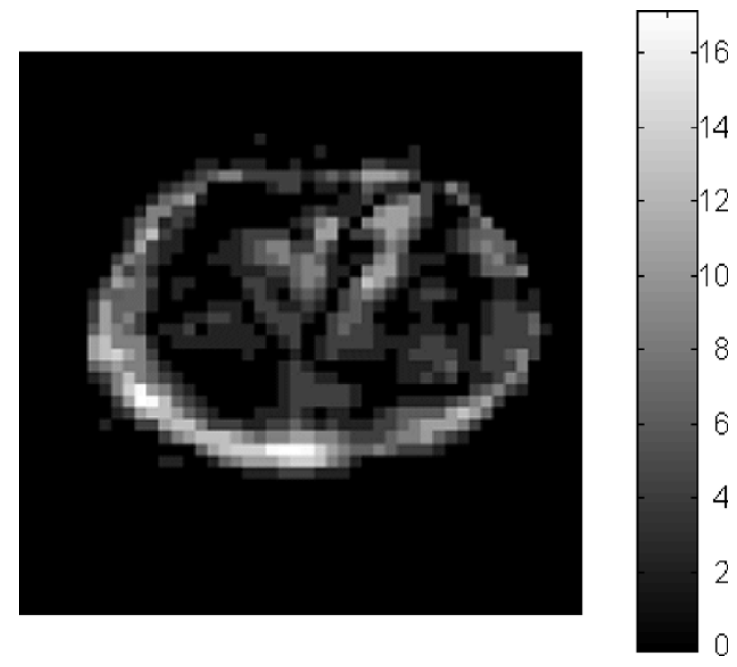

Fig. 13. The difference image for the activity reconstructions obtained by sourceless and transmission-based attenuation correction methods. The grayscale is given in the number of counts.

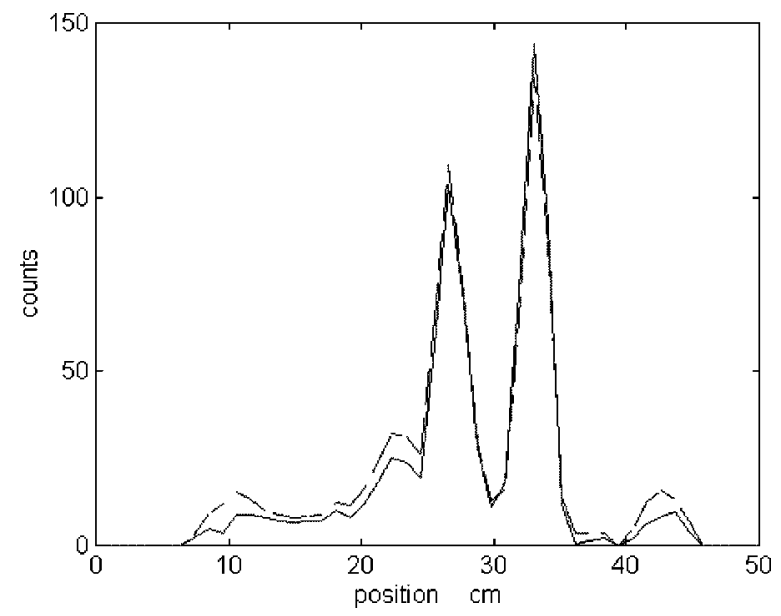

Fig. 14. The intensity profiles across the reconstructions of the activity image of the anthropomorphic torso phantom obtained with transmission-based attenuation correction (dashed lines) and sourceless attenuation correction.

cide well in both images. A certain error appears in the region of the background activity, where the image based on the estimated attenuation map has slightly lower values of intensity. To make

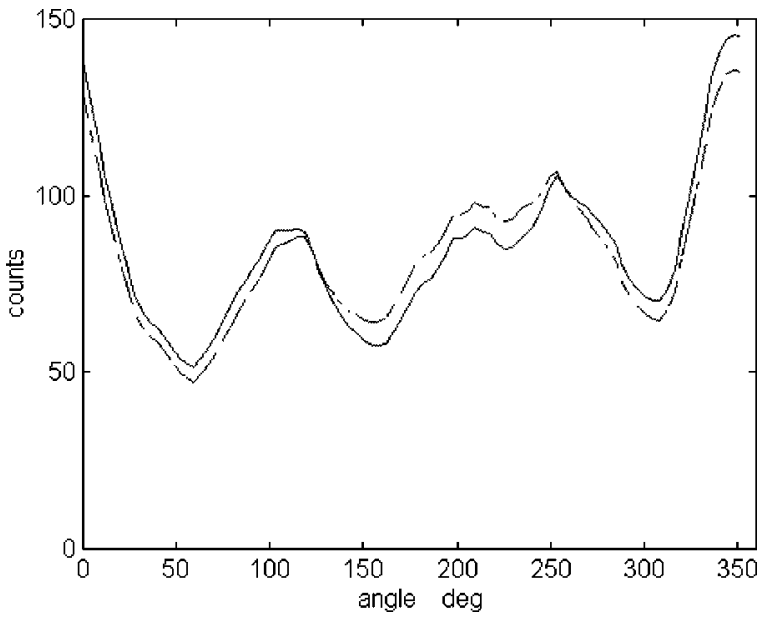

Fig. 15. Circumferential myocardial profiles of the reconstructions obtained with the transmission-based attenuation map (dashed lines) and the attenuation map estimated by the suggested approach.

a more detailed quantitative comparison in the heart region, we have computed the circumferential myocardial profiles, which are shown in Fig. 15. Here, we can see that the discrepancy in the heart region is not very large and lies in the range of $10 \%$. This means that the errors in the estimated attenuation map did not cause excessive deviations of the activity in the heart region. It is very likely that a further improvement of the image quality can be provided by compensating for scatter and the point-spread function while reconstructing the attenuation map. This may be a subject for the future research.

\section{CONCLUSION}

A new method based on discrete consistency conditions has been proposed for reconstruction of the attenuation map from emission projections. It has been shown that this type of condition can be written in the form of the orthogonal projection onto the complement to the column space of the matrix of the problem. A relation with the continuous consistency conditions has been established. In spite of an evident parallelism between the continuous and discrete cases, they provide different possibilities for solving the problem of reconstruction of the attenuation map. The main difference between them is of that 
kind existing between the analytical method of the filtered backprojection and the algebraic reconstruction technique. The discrete consistency conditions can easily be adapted to an arbitrary scanning configuration. In particular, they can be used in fully 3-D imaging and can be applied for both SPECT and PET with an arbitrary data acquisition geometry. The discrete conditions allow a relatively simple computer implementation by means of factorization of the matrix. In this paper, we have applied fast algorithms of the QR and Cholesky decompositions in combination with the Tikhonov regularization used for stabilizing the Newton method. Concerning the computer implementation, we remark that the QR and Cholesky factorizations are readily available in most mathematical software libraries. The corresponding algorithms have been described elsewhere (see, e.g., [20]) and are therefore not considered in this paper.

The algorithm has been evaluated with computer-generated and experimental data. Problems of 2-D parallel-beam and 3-D cone-beam SPECT cardiac imaging have been simulated. The results of simulations demonstrated stable reconstruction of the attenuation map with minimal a priori knowledge. In particular, nonnegativity of the attenuation map was taken into account, and in some experiments, a known support of the function was assumed. The latter permitted the number of unknowns to be considerably reduced in the problems with the high resolution of the image and in the fully 3-D problem. No crosstalk between the unknown source function and the reconstructed attenuation map was visible. An experiment with an anthropomorphic torso phantom showed promising results in reconstruction of the attenuation map of the thorax cross section characterized by relatively large variations of attenuation. Promising results have also been obtained in using the estimated attenuation maps for sourceless attenuation correction in computer simulations as well as in real data processing. At the same time, it should be pointed out that the discrete consistency conditions algorithm is not a method for simultaneous reconstruction of the activity image and the attenuation map. The primary purpose of the suggested method is to reconstruct the attenuation map from emission projections, whereas the source function $f_{\beta}$ can be seen as a sort of "byproduct" of the particular numerical implementation of the algorithm. After the attenuation map is obtained, the activity image can be found by other algorithms. This is illustrated in Section IV by several examples provided by applying the Tikhonov regularization method with the regularization parameter $\beta$ different from that of the algorithm for computing the attenuation map. Although we have considered a number of the most important numerical examples, a single publication cannot indeed cover all issues of studying the new approach. More work will be required in assessing the clinical applicability of the discrete consistency conditions. This future work should include not only evaluation of the suggested method with different attenuation correction algorithms, but also a detailed study of the influence of various physical factors, such as counting statistics, data acquisition geometry, scatter, point-spread function, and partial volume effect.

\section{ACKNOWLEDGMENT}

The author would like to thank Dr. F. Beekman and C. Kamphuis of Utrecht University Hospital for providing the anthropomorphic phantom data and the anonymous reviewers for their constructive comments on the evaluation of the method.

\section{REFERENCES}

[1] Y. Censor, D. Gustafson, A. Lent, and H. Tuy, "A new approach to the emission computerized tomography problem: Simultaneous calculation of attenuation and activity coefficients," IEEE Trans. Nucl. Sci., vol. 26, pp. 2275-2279, 1979.

[2] R. Kusaba, M. Nakajiama, K. Ogawa, S. Yuta, and T. Morozumi, "Attenuation correction method for single-photon emission CT without any additional data collection," Syst. Comput. Jpn., vol. 18, pp. 64-71, 1987.

[3] S. H. Manglos and T. M. Young, "Constrained IntraSPECT reconstruction from SPECT projections," Proc. IEEE Nucl. Sci. Symp. Med. Imag. Conf., vol. 3, pp. 1605-1609, 1993.

[4] A. Krol, S. H. Manglos, J. F. Bowsher, T. Young, D. A. Bassano, and F. D. Thomas, "Attenuation compensation in SPECT cardiac imaging using EM-IntraSPECT method,” J. Nucl. Med., vol. 36, p. 50P, 1995.

[5] J. Nuyts, P. Dupont, S. Stroobants, R. Benninck, L. Mortelmans, and P. Suetens, "Simultaneous maximum a posteriori reconstruction of attenuation and activity distributions from emission sinograms," IEEE Trans. Med. Imag., vol. 18, pp. 393-403, 1999.

[6] M. S. Kaplan, D. R. Haynor, and H. Vija, "A differential attenuation method for simultaneous estimation of SPECT activity and attenuation distributions," IEEE Trans. Nucl. Sci., vol. 46, pp. 535-541, 1999.

[7] V. Dicken, "A new approach toward simultaneous activity and attenuation reconstruction in emission tomography," Inv. Probl., vol. 15, pp. 931-960, 1999.

[8] A. V. Bronnikov and N. G. Preobrazhenskii, "Maximum entropy method in emission tomography of semitransparent media," Sov. Phys. Dokl., vol. 36, pp. 64-66, 1991 .

[9] A. V. Bronnikov, "Emission tomography of self-absorptive sources of radiation," Sov. Phys. Dokl., vol. 37, pp. 76-78, 1992.

[10] — "Degradation transform in tomography," Pattern Recognit. Lett., vol. 15, pp. 527-532, 1994.

[11] — , "Approximate reconstruction of attenuation map in SPECT imaging," IEEE Trans. Nucl. Sci., vol. 42, pp. 1483-1488, 1995.

[12] F. Natterer, "The identification problem in emission tomography," in Mathematical Aspects of Computerized Tomography. Lecture Notes in Medical Informatics, G. T. Herman and F. Natterer, Eds. New York: Springer-Verlag, 1981, vol. 8.

[13] — "Computerized tomography with unknown sources," SIAM J. Appl. Math., vol. 43, pp. 1201-1212, 1983.

[14] — "Determination of tissue attenuation in emission tomography of optically dense media," Inv. Probl., vol. 9, pp. 731-736, 1993.

[15] S. C. Moore, M. F. Kijewski, and S. P. Mueller, "A general approach to nonuniform attenuation correction using emission data alone," $\mathrm{J}$. Nucl. Med., vol. 38, p. 68p, 1997.

[16] A. Welch, R. Clack, F. Natterer, and G. T. Gullberg, "Toward accurate attenuation correction in SPECT without transmission measurements," IEEE Trans. Med. Imag., vol. 16, pp. 532-540, 1997.

[17] G. H. Golub and V. Pereyra, "The differentiation of pseudo-inverses and nonlinear least squares problems whose variables separate," SIAM J. Numer. Anal., vol. 10, pp. 413-432, 1973.

[18] A. V. Bronnikov, "Numerical solution of the identification problem for the attenuated Radon transform," Inv. Probl., vol. 15, pp. 1315-1324, 1999.

[19] F. J. Beekman, C. Kamphuis, B. F. Hutton, and P. P. van Rijk, "Half-fanbeam collimators combined with scanning point sources for simultaneous emission-transmission imaging," J. Nucl. Med., vol. 39, pp. 1996-2003, 1998.

[20] A. Bjorck, "Least squares methods," in Handbook of Numerical Analysis. Amsterdam: Elsevier, 1990, vol. 1, pp. 467-652. 